Ann. Génét. Sél. anim., I977, 9 (3), 275-282.

\title{
L'Aneuploïdie et la Polyploïdie chez les bovins dans les cultures de cellules bovines
}

\author{
E. P. CRIBIU et C.P. POPESCU \\ avec la collaboration technique de Jeannine Boscher \\ Laboratoive de Cytogénétique UNCEIA-INRA \\ Centre national de Recherches zootechniques \\ 78350 Jouy-en-Josas, (France)
}

\section{Résumé}

Une étude portant sur la fréquence des cellules péridiploïdes et polyploïdes a été entreprise, d'une part parmi $42 \mathrm{I}$ taureaux d'insémination artificielle appartenant à 5 races françaises : Charolaise, Maine-Anjou, Limousine, Normande et Frisonne, et d'autre part chez 5 lignées cellulaires embryonnaires reinales parmi lesquelles 3 étaient porteuses de la fusion centrique I/29.

Des différences hautement significatives entre les fréquences des cellules polyploïdes de chaque race bovine ont été observées.

\section{Introduction}

Le nombre de 6 o chromosomes chez Bos taurus $L$. a été précisé par de nombreux auteurs qui utilisaient soit la technique histologique (KRALLINGER (I927); MAKINO (I943), soit la technique du squash (MELANDER, I959), soit les cultures cellulaires (ChIARELli, I960). Cependant, on observe fréquemment, dans les cultures cellulaires à court et à long terme, une quantité, non négligeable, de métaphases comprenant un nombre de chromosomes pouvant varier de la quasi-haploïdie à la polyploïdie.

Dans le monde animal, l'aneuploïdie et la polyploïdie des cellules somatiques peuvent être induites selon FECHHEIMER (I972) par trois types de facteurs : physiques (radiation ionisantes, chocs thermiques), chimiques et biologiques. Mais il n'exclut pas que la fréquence de celles-ci puisse avoir un déterminisme génétique.

Notre étude qui rapporte des observations sur les leucocytes de taureaux normaux et sur des lignées cellulaires reinales normales et porteuses de la fusion centrique I/29 (Gustavsson I964) a pour but l'obtention de fréquences de référence pour la recherche d'anomalies types : mosaïques ou chimérismes. 


\section{Matériel et méthode}

\section{Les cultures de sang périphérique}

Pour chaque taureau, nous avons fait deux tubes de culture de sang entier selon la technique de DE GROUCHY et al, I964. Le sang a été distribué stérilement à raison de Io gouttes par tube de culture contenant : Io $\mathrm{ml}$ de Milieu TC I99, 20 p. Ioo de serum fœtal bovin, 0,02 $\mathrm{ml}$ Phytohemaglutinine $\mathrm{M}, 0,005 \mathrm{ml}$ de Phytohemaglutinine $\mathbf{P}$ et des antibiotiques (Ioo U.I./ml de Penicilline Streptomicine et IoO U.I. $/ \mathrm{ml}$ de Mycostatine). Les cultures ont ensuite été mises à l'étuve à $37^{\circ} \mathrm{C}$ pendant 66 heures. Une heure et demi avant l'arrêt $0,5 \mathrm{ml}$ de colchicine (concentration finale : $0,02 \mathrm{~g} / \mathrm{l}$ ) ont été ajoutés dans chaque tube. En ce qui concerne le traitement : le choc hypotonique a été effectué avec du citrate de sodium $(0,85 \mathrm{p}$. Ioo) et la fixation avec un mélange alcool absolu-acide acétique 3 : x. Pour chaque tube nous avons fait deux lames qui ont été séchées à la flamme (" air-drying ») puis colorées avec du Giemsa 4 p. roo.

\section{La péridiploïdie.}

L'étude porte sur 300 taureaux âgés de 2 ans environ, appartenant surtout à 2 races françaises : Frisons et Normands. Les chromosomes de cinq métaphases par animal, présentant une bonne dispersion et un degré moyen de spiralisation, ont été comptés directement au microscope à l'aide d'une chambre claire LEITz.

\section{La polyploïdie.}

Nous avons utilisé I2I taureaux âgés de 2 ans environ, appartenant à 5 races françaises : Charolais (30), Maine-Anjou (I2) Limousin (19), Normand (30) et Frisons (30) et compté pour chacun d'eux entre roo et 500 métaphases.

\section{Les cultures de Reins.}

Les cultures primaires ont été obtenues par trypsination de reins embryonnaires de veaux limousins, âgés de I à 3 mois, normaux et porteurs de la translocation robertsonienne I/29 (POPESCU, non publié) et cultivés pendant plusieurs mois dans les flacons Falcon. A l'occasion de chaque passage une petite quantité de cellules était distribuée dans des tubes à lamelles de type Leighton et traitée selon la méthode de DE Grouchy et Roubin (I965), légèrement modifiée. Cette technique a l'avantage de limiter au maximum les pertes chromosomiques dues au traitement car les cellules restent toujours collées à la lamelle.

\section{Résultats}

\section{La Péridiploïdie}

Le tableau I représente les fréquences de métaphases péridiploïdes trouvés dans une lignée de cellules embryonnaires de rein porteur de la fusion centrique I $/ 29$. 


\section{TABLEAU I}

Nombre (Nb) et fréquence (F.) des cellules péridiploïdes dans une lignée cellulaive reinale bovine (83), porteuse de la fusion centrique $I / 29$.

Number and frequency of peridiploid cells in a cell line of cattle kidney (83) with the I/29 centric fusion

\begin{tabular}{|c|c|c|c|c|c|c|c|c|c|}
\hline \multirow[b]{2}{*}{ Passages } & \multirow{2}{*}{\multicolumn{2}{|c|}{. . . }} & \multicolumn{7}{|c|}{ Nombre de chromosomes } \\
\hline & & & 56 & 57 & $5^{8}$ & 59 & 60 & $6 r$ & 62 \\
\hline $\mathrm{I}$ & $\begin{array}{l}\mathrm{Nb} \\
\mathrm{F} .\end{array}$ &. & $\begin{array}{c}o \\
0,00\end{array}$ & $\begin{array}{l}2 \\
2,10\end{array}$ & $\begin{array}{l}3 \\
3,16\end{array}$ & $\begin{array}{l}85 \\
89,47\end{array}$ & $\begin{array}{l}4 \\
4,2 \text { I }\end{array}$ & $\begin{array}{l}0 \\
0,00\end{array}$ & $\begin{array}{l}\mathrm{I} \\
\mathrm{I}, 05\end{array}$ \\
\hline II & $\begin{array}{l}\mathrm{Nb} \\
\mathrm{F} .\end{array}$ & $\begin{array}{l}\cdot . \\
\cdot .\end{array}$ & o & o & o & $\underline{4}$ & I & $\stackrel{0}{-}$ & o \\
\hline IV & $\begin{array}{l}\mathrm{Nb} \\
\mathrm{F} .\end{array}$ & .. & o & 3 & $\underline{3}$ & $\begin{array}{c}\text { I } \\
-\end{array}$ & I & o & o \\
\hline VI & $\begin{array}{l}\mathrm{Nb} \\
\mathrm{F} .\end{array}$ & $\cdot$ & - & $\underline{3}$ & 2 & I9 & o & o & $\stackrel{o}{-}$ \\
\hline Total & $\begin{array}{l}\mathrm{Nb} \\
\mathrm{F} .\end{array}$ & $\begin{array}{l}\cdot . \\
\cdot .\end{array}$ & $\begin{array}{l}0 \\
0,00\end{array}$ & $\begin{array}{l}8 \\
5,44\end{array}$ & $\begin{array}{l}8 \\
5,44\end{array}$ & $\begin{array}{l}\text { I } 24 \\
84,35\end{array}$ & $\begin{array}{l}6 \\
4,08\end{array}$ & $\begin{array}{l}0 \\
0,00\end{array}$ & $\begin{array}{l}\mathrm{I} \\
0,68\end{array}$ \\
\hline
\end{tabular}

Dans le tableau 2, nous avons mis les fréquences des cellules hypo et hyperdiploïdes calculées à partir des cultures de sang périphérique de 300 taureaux d'insémination.

\section{TABLEAU 2}

Fréquence des cellules péridiploïdes dans les cultures de leucocytes bovins normanx.

( $N . b$. nombre de cellules - F. fréquence des cellules en \%)

Frequency of peridiploid cells in normal cattle leucocyte cultures

\begin{tabular}{|c|c|c|c|c|c|c|c|c|c|c|}
\hline $\begin{array}{c}\text { Nombre de chro- } \\
\text { mosomes } \\
.\end{array}$ & 54 & 55 & $5^{6}$ & 57 & $5^{8}$ & 59 & 60 & $6 \mathrm{I}$ & 62 & 63 \\
\hline N.b. . . . . . . & 9 & I I & 22 & 45 & $6 \mathrm{r}$ & $x 6 x$ & III 8 & $5^{6}$ & $I_{4}$ & 3 \\
\hline $\mathrm{F} . \cdot \cdot \cdot$ & 0,60 & 0,73 & $\mathrm{I}, 47$ & 3,00 & 4,07 & ro,73 & 75,53 & 3,73 & 0,93 & 0,20 \\
\hline
\end{tabular}




\section{La polyploïdie}

Les fréquences de cellules polyploïdes détectées dans les 5 cultures de cellules reinales embryonnaires ont été représentées dans le tableau 3 .

\section{TABLEAU 3}

Fréquence des cellules polyploïdes dans 5 lignées bovines normales et porteuses de la fusion $I / 29$ $\left(83: 59, \mathrm{XX}(t: I-29) ; 136: 59, \mathrm{XX}(t: I-29) ; 3^{I I-I}: 59\right.$, XX $(t: I-29) ; 3^{I I-3}: 60, \mathrm{XX} ; 3^{I I-4}:$ $60, \mathrm{XX})$.

Frequency of polyploid cells in five cattle lines, normal and wearing I/29 fusion.

\begin{tabular}{|c|c|c|c|c|}
\hline $\begin{array}{l}\text { Nom de la } \\
\text { lignée }\end{array}$ & passages & $\begin{array}{l}\text { Nombre } \\
\text { de cellules } 2 \mathrm{~N}\end{array}$ & $\begin{array}{l}\text { Nombre } \\
\text { de cellules }{ }_{4} \mathrm{~N}\end{array}$ & $\begin{array}{l}\text { Fréquence } \\
\text { en } \%\end{array}$ \\
\hline 83 & $\begin{array}{l}\text { I } \\
\text { II } \\
\text { IV } \\
\text { VI }\end{array}$ & $\begin{array}{r}5250 \\
141 \\
119 \\
597\end{array}$ & $\begin{array}{r}79 \\
5 \\
7 \\
15\end{array}$ & $\begin{array}{l}I, 48 \\
3,42 \\
5,56 \\
2,45\end{array}$ \\
\hline r 36 & I & 885 & 40 & 4,32 \\
\hline $3 I I-I$ & I & 258 & $\mathbf{I}$ & 0,39 \\
\hline $3 I I-3$ & $\underset{\text { IV }}{\text { I }}$ & $\begin{array}{r}73 \\
668\end{array}$ & $\begin{array}{r}\mathbf{I} \\
\mathbf{1} 7\end{array}$ & $\begin{array}{l}1,35 \\
2,4^{8}\end{array}$ \\
\hline $3 \mathrm{Ir}-4$ & $\begin{array}{r}\text { I } \\
\text { IV }\end{array}$ & $\begin{array}{l}263 \\
28 I\end{array}$ & $\begin{array}{l}\text { I } \\
9\end{array}$ & $\begin{array}{l}0,38 \\
3,10\end{array}$ \\
\hline
\end{tabular}

Dans les cultures de sang entier, pour estimer la variation intra-raciale, nous avons fait un test d'homogénéité (tabl. 4) à l'intérieur de chaque race. Les $\chi^{2}$ trouvés ne se sont pas révélés significatifs au seuil de 5 p. roo.

Nous avons ensuite calculé les fréquences moyennes des cellulespolyplö̈es de chaque race (tabl. 4) afin de les comparer entre elles par un test d'analyse de variance. Pour cela, nous avons fait, tout d'abord, un test d'homogénéité des variances (Test de Bartlett) : $\chi^{2}=2$, oI pour 4 ddl $0,75>\mathrm{P}>0,50$, puis une transformation angulaire à l'aide des arcsinus.

L'analyse de variance (tabl. 5) a mis en évidence des différences hautement significatives entre les moyennes des fréquences des cellules polyploïdes des différentes races au seuil de I p. rooo.

Le test de Duncan a montré que seulement les moyennes des fréquences des cellules tétraploïdes des Charolais et des Maine-Anjou sont significativement différentes au seuil de 5 p. roo. 


\section{TABLEAU 4}

Fréquence moyenne des cellules polyploïdes et $\chi^{2} d^{\prime}$ hétérogénéité dans chaque race. Mean frequency of polyploid cells and heterogeneity $\chi^{2}$ in each breed

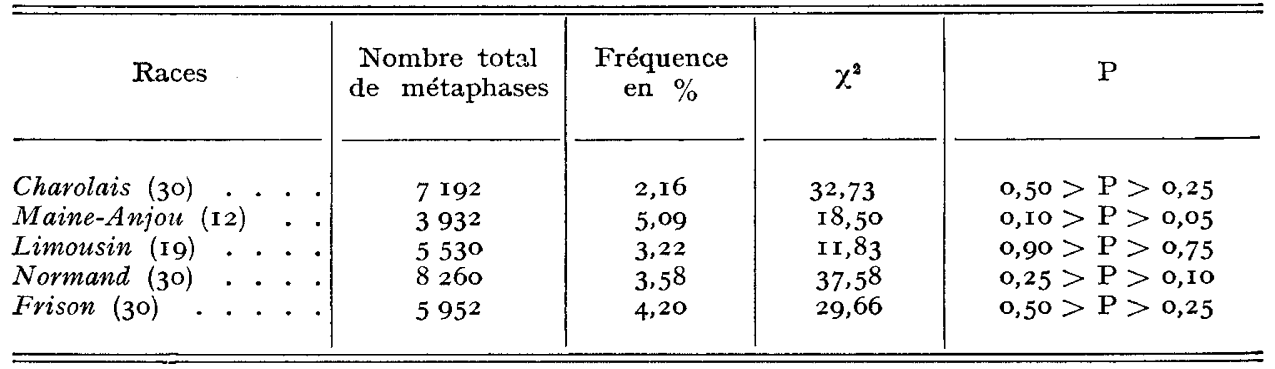

TABLEAU 5

Analyse de variance testant les différences entre les fréquences des 5 races bovines Analysis of variance for testing differences between 5 cattle breeds

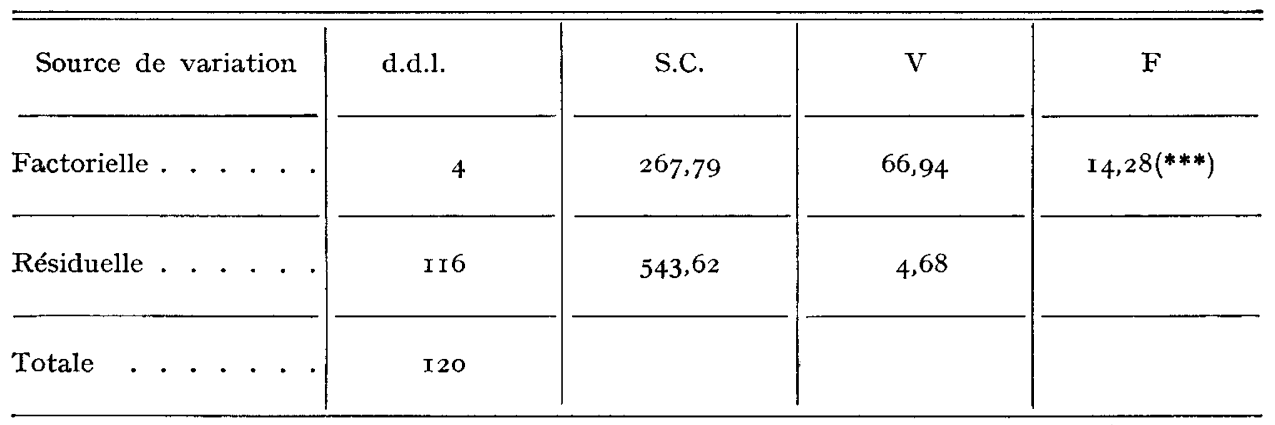

$(* * *) \mathrm{P}<0$, oor.

\section{Discussion}

Les résultats présentés dans les cultures de leucocytes et les cellules rénales, mettent en évidence, l'existence d'un nombre de cellules qui ne possèdent pas le nombre modale de chromosomes. Ces métaphases inhabituelles ont des nombres chromosomiques variant de l'hypodiploïdie à la tétraploïdie.

\section{Cellules hypodiplö̈des et hyperdiploïdes}

Deux hypothèses permettent d'expliquer la présence de telles cellules : les conditions de traitement et une non disjonction des chromosomes lors de l'anaphase.

Deux facteurs peuvent entraîner des pertes lors du traitement des cultures 
de leucocytes : un choc hypotonique trop violent qui conduit à l'éclatement des noyaux et à une dispersion beaucoup trop importante des métaphases et lors de l'étalement sur les lames un choc calorique trop intense. Pour les cellules hyperdiploïdes, le ou les chromosomes surnuméraires pourraient être venus d'une autre cellule. Cependant, cette possibilité est souvent à exclure car de telles cellules sont facilement identifiables en raison de la différence de spiralisation des chromosomes entre les deux métaphases.

Une non disjonction d'un chromosome pendant l'anaphase a pour conséquence, selon HAMERTon et al, (I965), la naissance de deux cellules filles dont l'une possède un chromosome en excès et l'autre un en moins.

En fait, à la lumière de nos résultats, nous pensons que ces deux phénomènes ont lieu dans les cultures de sang périphérique. Nous constatons, en effet, une différence importante entre les fréquences des métaphases à 59 chromosomes et à $6 \mathrm{I}$ chromosomes. Si nous admettons que la présence des cellules à $6 \mathrm{r}$ chromosomes est causée par une non-disjonction lors de l'anaphase de la cellule mère, nous pouvons donc, conclure que la fréquence de ce phénomène est de 3,73 p. Ioo. En conséquence, en l'absence d'une sélection quelconque, la différence enregistrée avec les cellules à 59 chromosomes (I0,73 p. IOO) est due aux pertes causées par le traitement lui-même.

Les cultures de cellules embryonnaires rénales, nous apportent une confirmation de notre hypothèse. Pendant le traitement, les cellules restent collées sur la lamelle, ce qui entraîne peu de pertes dues aux manipulations techniques. Nous constatons, en effet, que les fréquences des cellules hypo et hyper-diploïdes (tabl. I) au cours du premier passage sont du même ordre de grandeur : 3,I6 p. Ioo pour les métaphases à 58 chromosomes et $4,2 \mathrm{I}$ p. Ioo pour les cellules ayant 60 chromosomes.

\section{Cellules tétraploïdes}

Trois hypothèses expliquent l'origine des cellules tétraploïdes dans les cultures cellulaires : les endomitoses (Genest, I967; Sinha, I967, I973), la fusion de deux cellules (Sinha, I967 et DEAven et KREISINGER, I97I) et une caryocinèse qui n'est pas suivi par une cytocinèse.

Les différences significatives enregistrées entre les fréquences des leucocytes polyploïdes dans les différentes races bovines semblent confirmer l'opinion exprimée par FECHHEIMER, (I972) sur un déterminisme génétique de la formation de telles cellules. Nous en trouvons de nombreuses preuves dans la littérature, GLook et STEIger (I954) décrivent un allèle récessif qui bloque la formation du fuseau dans les cellules de larves de Drosophila hydei; Dans l'anémie de Fanconi chez l'homme les lymphocytes de certains patients montrent une proportion élevée d'endomitoses (Bloom et al, (I966); Emery et al., (I969); chez les dindes, un allèle autosomal récessif provoque l'apparition de globules rouges binuclés qui semblent avoir un mode d'origine différent (Bloom et al. I970); Popescu (I968) chez les taureaux " culards " Charolais trouve des pourcentages de leucocytes polyploïdes de I7 à $20 \mathrm{p}$. Ioo pour les homozygotes et de II à I4 p. Ioo pour les hétérozygotes et enfin, HERzOG et HöHN (I97I) chez des bovins atteints de troubles du système nerveux centrales mettent en évidence l'existence d'une polyploïdie élevée. ZARTMAN et FECHHEIMER (I967) en comparant le taux de cellules polyploïdes leucocytaires dans les lignées de bovins, trouvent des différences presque significatives $0, \mathrm{I}>\mathrm{P}>0,05$ entre elles. 


\section{Conclusion}

Nous possédons des fréquences de référence pour la détection de mosaïque aneuploïde/diploïde parmi les taureaux d'insémination avec les résultats présentés dans cette étude.

Il conviendra, en effet, d'être prudent dans les cas de mosaïques faibles où la fréquence de l'aneuploïde est inférieure à Io p. Ioo, avant de conclure à une anomalie. Seules les techniques de marquage $G, R$ ou $Q$ pourraient permettre de trancher dans ces cas.

Reçu pour publication en juillet 1977.

\section{Summary}

\section{Somatic aneuploidy and polyploidy in bovine cell cultures}

A study of somatic peridiploid and polyploid cell frequencies was conducted using cultures lymphocytes from 421 A.I. bulls of 5 French breeds: Charolais, Maine-Anjou, Limousin, Norman and Friesian, moreover 5 bovine kidney cell lines. Difference in the distribution of polyploid lymphocytes between breeds had been tested.

No significant differences are found within groups. However significant differences among the five breeds are observed.

\section{Références bibliographiques}

Bloom G. F., Warner S., Gerald P. S., Diamond L. K., ig66. Chromosomes abnormalities in constitutional aplastic anemia. New Engl. J. Med. 274, 8.

Bloom S. E., Buss E. G., Strother G. J., 197o. Cytological and cytophotometric analysis of binucleated red blood cell mutants (bn) in turkeys (Meleagris gallopavo). Genetics, 65, 57.

Chiarelli B., De Carli L., Nuzzo F., I96o. Analisi morfometrica dei chromosome di Bos taunus L. Caryologia, 13, 766-772.

Deaven L. L., Kreizinger J. D., I97I. Cell fusion as a mechanism for the origin of polyploid cells in vitro. Experientia, 27, 3II-312.

De Grouchy J., Roubin M., Passage E., I964. Microtechnique pour l'étude des chromosomes humains à partir d'une culture de leucocytes sanguins. Ann. Génét. 7, 45.

De Grouchy J., Roubin M., 1965. Une technique d'étude des chromosomes humains à partir d'une culture de fibroblastes. Ann. Génét., 8 : го5-106.

Emery A. E. M., Perkins J., Trmson J., I969. Clinical and chromosome studies in Fanconi's aplastic anemia. J. med. Genet. 6, 28.

Fechheimer N. S., 1972. Causal basis of chromosome abnormalities. J. Reprod. Fert., (Suppl.) 15, 79-98.

Genest P., 1967. Observation d'endoreduplications dans des microcultures du sang d'individus normaux ou atteints de troubles variés. Hypothèse d'une sensibilité constitutionnelle des leucocytes à la Phytohemagglutinine. Rev. can. Biol., 26, I9I-I97.

Gloor H., Staiger M., 1954. Lethal polyploidy - a polyploid gene in Drosophila hydei. J. Heved. 45, 289.

Gustavsson I., RockBoRn G., 1964. Chromosome abnormality in three cases of lymphatic leukaemia in cattle. Nature 203, 990.

Hamerton J. L., Tailor A. I., Angell R., Mcguire V. M., I965. Chromosome investigations of a small isolated human population: chromosome abnormalities and distribution of chromosome counts according to age and sex among population of Tristan da Cunha. Nature, 206: I 232.

Herzog A., HöHn N., 1971. Zytogenetische Befunde Bei Angeborenen Anomalien des Zentralnervensystems des Rindes. Ann Génét. Sél. anim., 3, 225-234. 
KRALlinger H., I927. Uber dei Chromosomanzahl beim Rinde sowie einige all-gemeine Bermerkungen über die Chromosomenforschung in der Säugetierklasse. Verh. anat. Ges. (Jena), 1927 Kiel, in Anat. Ang., Suppl., 63, 209-2 I4.

Makino S., I943. Chromosomes of domestic cattle and Buffalo. Botany Zool. Tokyo II, 29-3I. Melander Y., I959. The mitotic chromosomes of some cavicorn mammals, Bos taurus L. Bison bonasus $L$. and Ovis aries $L$. Hereditas, 45, 649-664.

Popescu C. P., r968. Observations cytogénétiques chez les bovins Charolais normaux et culards. Ann. Génét., 11, 262-264.

SrNhA A. K., r967. Spontaneous occurence of tetraploidy and near-haploidy in mammalian peripheral blood. Exptl. cell Res., 47: 443.

Srnha A. K., Kakatr S., Pathak S., 1973. Extent of ploidy in mammalian marrow and peripheral blood cells. Caryologia, 26, 333-345.

Zartman D. M., Fechineimer N. S., 1967. Somatic aneuploidy and polyploidy in inbred and line cross cattle. J. Anim. Sci. 26, 678 . 